\title{
Erratum: Three-dimensional quasistatic model for high brightness beam dynamics simulation [Phys. Rev. ST Accel. Beams 9, 044204 (2006)]
}

Ji Qiang, Steve Lidia, Robert D. Ryne, and Cecile Limborg-Deprey

(Received 30 November 2007; published 12 December 2007)

DOI: $10.1103 /$ PhysRevSTAB.10.129901

PACS numbers: 29.27.Bd, 99.10.Cd

There is a typographical error in Eq. (16) of Ref. [1]. The correct formula is

$$
\begin{aligned}
\iiint \frac{1}{\sqrt{x^{2}+y^{2}+z^{2}}} d x d y d z= & y z \ln \left(x+\sqrt{x^{2}+y^{2}+z^{2}}\right)+x z \ln \left(y+\sqrt{x^{2}+y^{2}+z^{2}}\right)+x y \ln \left(z+\sqrt{x^{2}+y^{2}+z^{2}}\right) \\
& -\frac{1}{4} i z^{2} \ln \left(\frac{-8 i x^{2}+8(y-i z) z-8 i x \sqrt{x^{2}+y^{2}+z^{2}}}{x^{2}(y-i z) z^{2}}\right) \\
& +\frac{1}{4} i z^{2} \ln \left(\frac{8 i x^{2}+8(y+i z) z+8 i x \sqrt{x^{2}+y^{2}+z^{2}}}{x^{2}(y+i z) z^{2}}\right) \\
& +\frac{1}{4} i x^{2} \ln \left(\frac{8\left(x^{2}-i x y+z\left(z+\sqrt{x^{2}+y^{2}+z^{2}}\right)\right)}{x^{2}(x-i y) z^{2}}\right) \\
& -\frac{1}{4} i x^{2} \ln \left(\frac{8\left(x^{2}+i x y+z\left(z+\sqrt{x^{2}+y^{2}+z^{2}}\right)\right)}{x^{2}(x+i y) z^{2}}\right) \\
& -\frac{1}{4} i y^{2} \ln \left(\frac{2 x y-2 i\left(y^{2}+z\left(z+\sqrt{x^{2}+y^{2}+z^{2}}\right)\right)}{y(x-i y) z^{2}}\right) \\
& +\frac{1}{4} i y^{2} \ln \left(\frac{2 x y+2 i\left(y^{2}+z\left(z+\sqrt{x^{2}+y^{2}+z^{2}}\right)\right)}{y(x+i y) z^{2}}\right) .
\end{aligned}
$$

Here, the terms that will not contribute to the electric potential are not included. A simpler form of the integral from a new version of MATHEMATICA [2] is

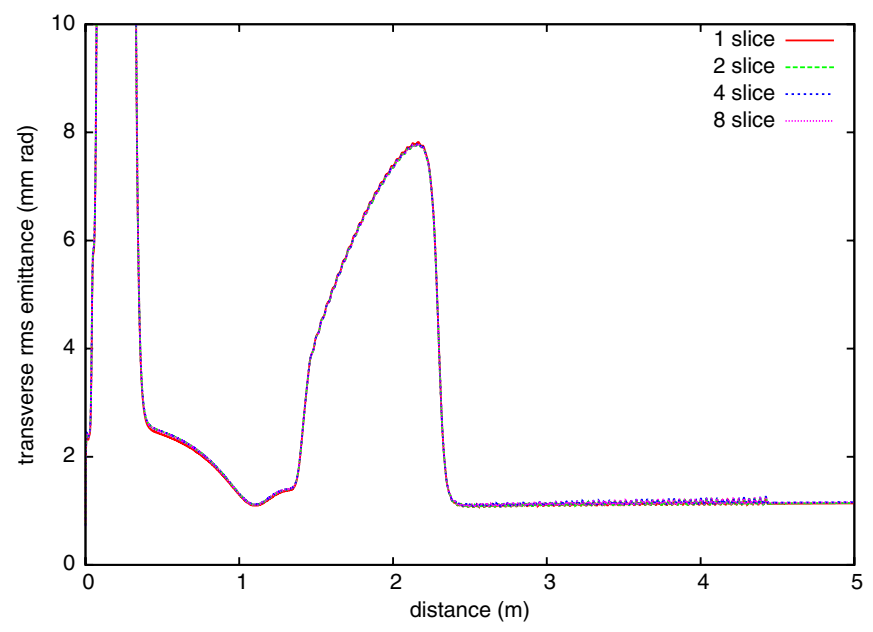

FIG. 1. (Color) Transverse normalized rms emittance as a function of distance using a one slice, a two slice, a four slice, and an eight slice model of the beam. 


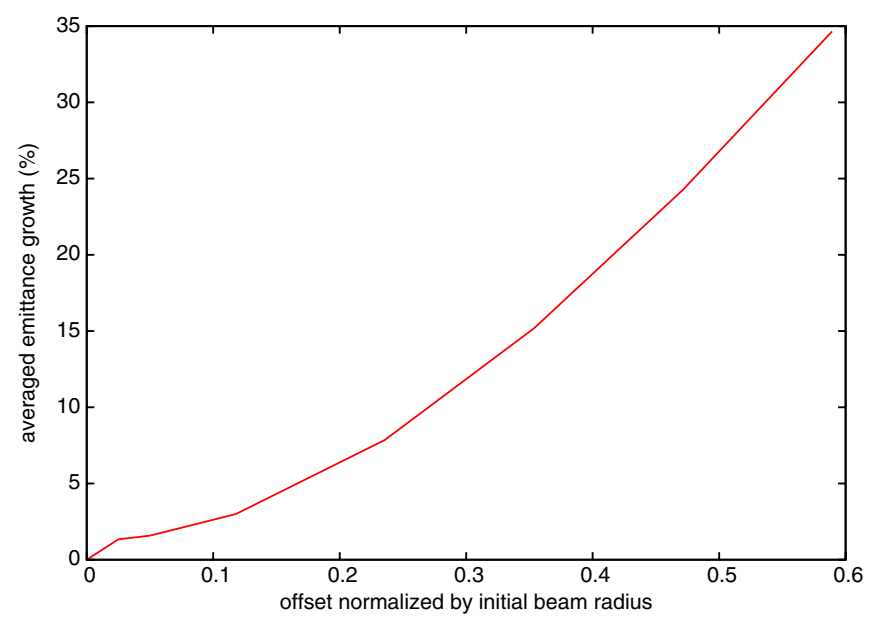

FIG. 2. (Color) Emittance growth at the end of the injector as a function of initial misalignment of the beam.

$$
\begin{aligned}
\iiint \frac{1}{\sqrt{x^{2}+y^{2}+z^{2}}} d x d y d z= & -\frac{z^{2}}{2} \arctan \left(\frac{x y}{z \sqrt{x^{2}+y^{2}+z^{2}}}\right)-\frac{y^{2}}{2} \arctan \left(\frac{x z}{y \sqrt{x^{2}+y^{2}+z^{2}}}\right)-\frac{x^{2}}{2} \arctan \left(\frac{y z}{x \sqrt{x^{2}+y^{2}+z^{2}}}\right) \\
& +y z \ln \left(x+\sqrt{x^{2}+y^{2}+z^{2}}\right)+x z \ln \left(y+\sqrt{x^{2}+y^{2}+z^{2}}\right)+x y \ln \left(z+\sqrt{x^{2}+y^{2}+z^{2}}\right) .
\end{aligned}
$$

There was also an error in our code that affected the multislice simulation results of the Linac Coherent Light Source (LCLS). The LCLS example was rerun using one, two, four, and eight slices. Figure 1 shows the normalized transverse rms emittances as a function of distance using a different number of slices. It appears that, in this example, the effect of energy spread is small and a single slice is sufficient. The final emittance at the end of $5.0 \mathrm{~m}$ is about $1.1 \mathrm{~mm}$ mrad. In some other applications, such as a high acceleration gradient DC gun proposed at PSI, it is found that a multiple slice model is needed in order to accurately calculate the space-charge forces in the rest frame [3].

The emittance growth at the end of the injector as a function of initial normalized misalignment of the beam was also rerun using the new version of the code. The updated results are shown in Fig. 2. The final emittance growth is smaller than the previous calculation. However, it still suggests that the initial misalignment of the beam should be controlled within 0.2 to $0.3 \sigma$ in order to keep the average emittance growth below the $10 \%$ level.

\section{ACKNOWLEDGMENTS}

We would like to thank Dr. L. M. Young for pointing out the typo in Eq. (16) and a simpler form from the new version of MATHEMATICA. This work was supported by the U.S. Department of Energy under Contract No. DE-AC02-05CH11231.

[1] J. Qiang, S. Lidia, R. D. Ryne, and C. Limborg-Deprey, Phys. Rev. ST Accel. Beams 9, 044204 (2006).

[2] http://www.wolfram.com/.

[3] A. Adelmann et al., in Proceedings of PAC07 Conference, Albuquerque, 2007, p. 785. 